\title{
MIN-MAX MODEL PREDICTIVE CONTROL AS A QUADRATIC PROGRAM
}

\author{
D. Muñoz de la Peña T. Alamo D.R. Ramírez \\ E.F. Camacho
}

\author{
Dep. de Ingeniería de Sistemas y Automática, Universidad \\ de Sevilla. Spain.
}

\begin{abstract}
This paper deals with the implementation of min-max model predictive control for constrained linear systems with bounded additive uncertainties and quadratic cost functions. This type of controller has been shown to be a continuous piecewise affine function of the state vector by geometrical methods. However, no algorithm for computing the explicit solution has been given. In this paper, we show that the min-max optimization problem can be expressed as a multi-parametric quadratic program, and so, the explicit form of the controller may be determined by standard multi-parametric techniques. Copyright ${ }^{\circledR} 2005$ IFAC.
\end{abstract}

Keywords: Predictive control, Robust control, Optimization devices, Uncertain systems, Constraints

\section{INTRODUCTION}

Model predictive control (MPC) is one of the control techniques that is able to cope with model uncertainties in an explicit way (Camacho and Bordóns, 2004). One approach used in MPC when uncertainties are present, is to minimize the objective function for the worst possible case. This strategy is known as min-max and was originally proposed in (H.S.Witsenhausen, 1968) in the context of robust receding control. In robust MPC the problem was first tackled in (P.J.Campo and Morari, 1987). In this paper we consider bounded additive uncertainties. For other approaches see (Kothare et al., 1996; Y.J.Wang and Rawlings, 2003; J.H.Lee and Yu, 1997; Bemporad et al., 2003) and the references therein.

Min-max MPC schemes can be classified in open loop and feedback min-max controllers (see (Mayne et al., 2000)). Feedback min-max MPC obtains a sequence of feedback control laws that minimizes the worst case cost, while assuring robust constraint handling. It requires the so- lution of a very high dimensional problem that makes its practical implementation very hard (see (Scokaert and Mayne, 1998)). For cost functions based on $\|.\|_{\infty}$ and $\|.\|_{1}$ norm, the explicit solution has been obtained (see (Bemporad et al., 2003; E.C.Kerrigan and Maciejowski, 2004)). This result has not been extended to quadratic cost functions although approximate solutions have been given under an stochastic approach in (Sakizlis et al., 2004).

Open loop min-max MPC obtains a single control input sequence that minimizes the worst case cost (see (P.J.Campo and Morari, 1987; Allwright and Papavasiliou, 1992)). This formulation is conservative and underestimates the set of feasible input trajectories. The solution proposed in the literature is to minimize a sequence of control corrections efforts to a given linear feedback stabilizing control law for the nominal plant. In this way, some kind of feedback is introduced in the prediction without increasing the computational effort (see (Bemporad, 1998; Alamo et al., 2003; Löfberg, 2003)). 
In this paper we consider the constrained open loop min-max MPC with linear feedback control law and quadratic cost functions. For these controllers, the size of the optimization problem is exponential on the prediction horizon. Suboptimal approaches with polynomial complexity are given in (Alamo et al., 2003; Löfberg, 2003).

Recently, the piecewise linearity of this class of min-max MPC has been proved by geometrical methods in (Ramírez and Camacho, 2001). However no algorithm has been given for determining the solution. In this paper, we show that the minmax optimization problem can be expressed as a multi-parametric quadratic problem (mp-QP) so the piecewise affine nature of the feedback control law is proven through Karush-Kuhn-Tucker optimality conditions. It is noteworthy to note that the resulting mp-QP problem is not equivalent to the one that appears in nominal MPC although it has been treated in recent multi-parametric works (see (Tøndel et al., 2003)).

The paper is organized as follows: Section 2 defines the control problem to be solved. Section 3 shows how to transform min-max MPC into an $\mathrm{mp}-\mathrm{QP}$ problem. An example is given in section 4 while some concluding remarks are given in Section 5.

\section{PROBLEM FORMULATION}

Consider the discrete invariant time linear system with bounded uncertainties

$$
x_{k+1}=A x_{k}+B u_{k}+D w_{k},
$$

where $x_{k} \in \Re^{n_{x}}$ is the state, $u_{k} \in \Re^{n_{u}}$ is the control input, and $w_{k} \in \Re^{n_{w}}$ is the uncertainty that is supposed to be bounded, that is $w_{k} \in W$ where $W$ is a closed polyhedron that contains the origin.

The control input is given by $u_{k}=K x_{k}+v_{k}$, where $K$ is chosen in order to achieve some desired property for the non constrained problem such as stability or LQR optimality (see (Bemporad, 1998; Alamo et al., 2003; Löfberg, 2003)). The MPC controller will compute the optimal sequence of correction control inputs $v_{k}$. The dynamics of the system can be rewritten as

$$
\begin{aligned}
& x_{k+1}=A_{K} x_{k}+B v_{k}+D w_{k}, \\
& A_{K}=(A+B K) .
\end{aligned}
$$

The objective function is defined as

$$
V(x, \mathbf{v}, \mathbf{w})=\sum_{j=0}^{N-1}\left[x_{j}^{T} Q x_{j}+u_{j}^{T} R u_{j}\right]+x_{N}^{T} P x_{N},
$$

with $Q>0, P>0$ and $R \geq 0$.

The initial state is $x_{0}=x$. Vector $\mathbf{v}=$ $\left\{v_{0}, v_{1}, \ldots, v_{N-1}\right\}$ is the sequence of correction control inputs, and $\mathbf{w}=\left\{w_{0}, w_{1}, \ldots, w_{N-1}\right\}$ represents a possible sequence of input disturbances to the system.

Variables $x_{j}$ and $u_{j}$ are the predicted state and control input respectively and are given by

$$
\begin{aligned}
& x_{j}=A_{K}^{j} x+\sum_{i=1}^{j} A_{K}^{i-1} B v_{j-i}+\sum_{i=1}^{j} A_{K}^{i-1} D w_{j-i}, \\
& u_{j}=K x_{j}+v_{j} .
\end{aligned}
$$

We consider state and input constraints $x_{k} \in X$ and $u_{k} \in U$ where $X$ and $U$ are polyhedral sets. In order to achieve stability, a polyhedral terminal region constraint, $x_{N} \in \Omega$, will also be taken into account. Note that in this formulation, any invariant time linear constraint can be taken into account, as for example operational constraints of the form

$$
O_{k} u_{k} \leq L_{k} x_{k}+o_{k}
$$

For simplicity on the notation, only state and input constraints are considered.

The min-max constrained predictive controller results in the solution of the following optimization problem denoted $P(x)$

$$
J^{*}(x)=\min _{\mathbf{v}} \max _{\mathbf{w} \in W_{N}} V(x, \mathbf{v}, \mathbf{w}),
$$

subject to:

$$
\begin{aligned}
& x_{j}(x, \mathbf{v}, \mathbf{w}) \in X, \quad \forall \mathbf{w} \in W_{N}, \quad j=0 \ldots N, \\
& x_{N}(x, \mathbf{v}, \mathbf{w}) \in \Omega, \quad \forall \mathbf{w} \in W_{N}, \\
& u_{j}(x, \mathbf{v}, \mathbf{w}) \in U, \quad \forall \mathbf{w} \in W_{N}, \quad j=0 \ldots N-1 .
\end{aligned}
$$

where $W_{N}$ denotes the set of possible disturbance sequences of length $N$ :

$$
W_{N}=\left\{\mathbf{w}=\left\{w_{0} \ldots w_{N-1}\right\} \mid w_{i} \in W, \forall i\right\} .
$$

This optimization problem is solved at each sample instant. An optimal vector of control correction signals $\mathbf{v}^{*}$ is obtained and the control input $u_{0}=K x+v_{0}^{*}=K_{M P C}(x)$ is applied. All this is done in a receding horizon manner, see (Camacho and Bordóns, 2004).

An appropriate choice of the cost function and the terminal region may assure robust convergence to a bounded set and robust constraint fulfillment from any feasible initial state (see Theorem 1, (Alamo et al., 2003)), namely

- If $x \in \Omega$ then $A_{K} x+D w \in \Omega, \forall w \in W$.

- If $x \in \Omega$ then $K x \in U$.

- $P-A_{K}^{T} P A_{K}>Q+K^{T} R K$.

The stability of $A_{K}=A+B K$ guarantees the existence of a positive definite matrix $P$ that satisfies the third condition.

\subsection{Min-max MPC computation}

In order to implement the proposed controller, problem (3) subject to constraints (4) has to be 
solved at each sampling time. Let us define the feasible set $S_{F}$ as the pairs $(x, \mathbf{v})$ which satisfy constraints (4). Taking into account (2), when $X$, $\Omega$ and $U$ are polyhedral regions, matrices $F, G, m$ and $M$ can be found such that the feasible set $S_{F}$ can be expressed as

$$
S_{F}=\left\{(x, \mathbf{v}) \mid F x+G \mathbf{v} \leq m+M \mathbf{w}, \forall \mathbf{w} \in W_{N}\right\} .
$$

For the system described (i.e. linear systems and additive uncertainties) it is possible to reduce the number of constraints defining the feasible set $S_{F}$. It can be seen that definition (5) is equivalent to

$$
S_{F}=\{(x, \mathbf{v}) \mid F x+G \mathbf{v} \leq d\},
$$

where $d$ is a vector such that its $i$-th entry satisfies

$$
d_{i}=m_{i}+\min _{\mathbf{w} \in W_{N}} M_{i} \mathbf{w},
$$

and $m_{i}$ and $M_{i}$ are the $i$-th element and row of vector $m$ and matrix $M$ respectively.

The cost function, $V(x, \mathbf{v}, \mathbf{w})$, is a quadratic function of $x, \mathbf{v}$ and $\mathbf{w}$. That is, taking into account (2), matrices $H_{x}, H_{v}$ and $H_{w}$ can be found in such a way that

$$
V(x, \mathbf{v}, \mathbf{w})=\left\|H_{x} x+H_{v} \mathbf{v}+H_{w} \mathbf{w}\right\|_{2}^{2} .
$$

The cost function is a convex function on $x, \mathbf{v}$ and $\mathbf{w}$. The maximum of a convex function always lays in the boundary of the feasible region. Thus, the maximum in the future possible uncertainty trajectories can be obtained evaluating the cost function at the set of vertices of the hypercube $W_{N}$, denoted by $\mathcal{V}\left(W_{N}\right)$ (see (Bazaraa and Shetty, 1979)). That is,

$$
\max _{\mathbf{w} \in W_{N}} V(x, \mathbf{v}, \mathbf{w})=\max _{\mathbf{w} \in \mathcal{V}\left(W_{N}\right)} V(x, \mathbf{v}, \mathbf{w}) .
$$

We conclude that $P_{N}(x)$ can be rewritten as

$$
\begin{gathered}
J^{*}(x)=\min _{\mathbf{v}} \max _{\mathbf{w} \in \mathcal{V}\left(W_{N}\right)}\left\|H_{x} x+H_{v}, \mathbf{v}+H_{w} \mathbf{w}\right\|_{2}^{2} \\
\text { s.t. } \quad F x+G \mathbf{v} \leq d .
\end{gathered}
$$

The complexity of this optimization problem is very high. The max function is convex but its evaluation requires the computation of the cost function for each possible vertex of $W_{N}$. The number of these vertices is exponential with the prediction horizon $N$.

\section{MIN-MAX AS A QUADRATIC PROGRAMM}

It has been shown by geometrical methods (see (Ramírez and Camacho, 2001)) that a min-max MPC with a quadratic objective function and linear constraints turns out to be a piecewise affine function of the state. We show in this section that the min-max problem (3) can be expressed as a multi-parametric quadratic programming problem and that the explicit min-max MPC controller can be obtained using multi-parametric techniques.

If $V(x, \mathbf{v}, 0)$ (the part of the cost function that does not depend on the uncertainty) is added and subtracted to the cost function $V(x, \mathbf{v}, \mathbf{w})$ the min-max problem can be expressed as

$$
J^{*}(x)=\min _{\mathbf{v}, \gamma} V(x, \mathbf{v}, 0)+
$$

subject to

$$
\begin{aligned}
& F x+G \mathbf{v} \leq d, \\
& \quad \geq V(x, \mathbf{v}, \mathbf{w})-V(x, \mathbf{v}, 0), \quad \forall \mathbf{w} \in \mathcal{V}\left(W_{N}\right),
\end{aligned}
$$

with

$V(x, \mathbf{v}, \mathbf{w})-V(x, \mathbf{v}, 0)=\mathbf{w}^{T} H_{w}^{T} H_{w} \mathbf{w}+2 \mathbf{w}^{T} H_{w}^{T}\left(H_{x} x+H_{v} \mathbf{v}\right)$.

These constraints are all affine on $x$ and $\mathbf{v}$, however, it is important to remark that each one of them, one for each vertex of $W_{N}$, is defined by a different gain on $\mathbf{v}$ that depends on the the vertex $\left(2 \mathbf{w}^{T} H_{w}^{T} H_{v}\right)$, so they can not be reduced in the same way as the constraints (4) (although standard constraint reduction techniques may be applied) and an exponential number of constraints with the prediction horizon are posed.

In order to obtain an equivalent problem in which the functional does not depends on the state vector, we introduce the following variable change

$$
\mathbf{z} \triangleq \mathbf{v}+\left[H_{v}^{T} H_{v}\right]^{-1} H_{v}^{T} H_{x} x,
$$

then, the min-max problem defined in $(3), P(x)$, is equivalent to

$$
J^{*}(x)=x^{T} Y x+\min _{\mathbf{z}, \gamma} \frac{1}{2} \mathbf{z}^{T} H \mathbf{z}+
$$

subject to

$$
\begin{array}{r}
G_{m} \mathbf{z}+g_{m} \leq W_{m}+S_{m} x, \\
G_{c} \mathbf{z} \leq W_{c}+S_{c} x .
\end{array}
$$

The positive definiteness of $R$ assures that $H$ is positive definite $\left(H=2 H_{v}^{T} H_{v} \succ 0\right)$. The linear inequalities described by matrices $G_{m}, g_{m}, W_{m}$ and $S_{m}$ correspond to the maximization of the functional. The number of these inequalities, as the number of possible vertices, is exponential with the prediction horizon $N$. The inequalities defined by $G_{c}, W_{c}$ and $S_{c}$ represent the robust constraints of the problem. The matrices that define $(7),(8)$ and (9) can be obtained from the problem parameters.

It is important to note, that the functional is still strictly convex because $H \succ 0$, and corresponds to the maximum of a set of linear functions. This means that the optimization problem has an unique optimizer $\mathbf{z}^{*}$, ${ }^{*}$ and dual degeneracy cannot take place (see (Bazaraa and Shetty, 1979)). Also, in the optimum there is always at least 
one constraint out of $G_{m}, g_{m}, W_{m}$ and $S_{m}$ that is satisfied in a tight way, this is, is satisfied with the equality sign, as for any input correction trajectory $\mathbf{v}$, there is at least a vertex where the function takes the maximum value for all possible uncertainties.

\subsection{Application of Multi-Parametric Programming to Min-Max}

Multi-parametric quadratic (mp-QP) problems have been studied in the literature in the MPC context (see (Bemporad et al., 2002; Mayne and Rakovic, 2002; Seron et al., 2002)). However, the $\mathrm{mp}-\mathrm{QP}$ problem addressed in those references is different from the one posed in min-max MPC. The mp-QP problem posed in a nominal MPC controller is defined by a definite positive matrix, and therefore invertible. In min-max MPC, the auxiliary variable does not take part in the quadratic cost and so the resulting matrix is not invertible. The algorithm presented in (Tøndel et al., 2003) deals with semi-definite positive mpQp programs and can be applied to the problem presented here.

The active constraints that define the solution in a given state vector, can be distinguished in those corresponding to the original constraints of the system, and those corresponding to the evaluation of the maximum.

Definition 1. Given a state vector $x$ and the optimal solution pair $\mathbf{z}^{*}$ and ${ }^{*}$, we define the active vertices constraints set $I$, the set of constraints out of (8) which satisfy

$$
\tilde{G}_{m} \mathbf{z}^{*}+\tilde{g}_{m}{ }^{*}-\tilde{W}_{m}-\tilde{S}_{m} x=0 .
$$

Definition 2. Given a state vector $x$ and the optimal solution pair $\mathbf{z}^{*}$ and ${ }^{*}$, we define the active constraints set $A$, the set of constraints out of (9) which satisfy

$$
\tilde{G}_{c} \mathbf{z}^{*}-\tilde{W}_{c}-\tilde{S}_{c} x=0 .
$$

The active vertices constraints set of a solution defines the vertices in which the max function attains the maximum while the active constraints set defines the constraints of $P(x)$ which are satisfied in a tight way for the optimal solution in a given state vector.

\subsection{Degeneracy}

Degeneracy is a key issue in multi-parametric optimization. The optimum vector of lagrange multipliers may be not uniquely defined, this is known as primal degeneracy). In this case, the optimum
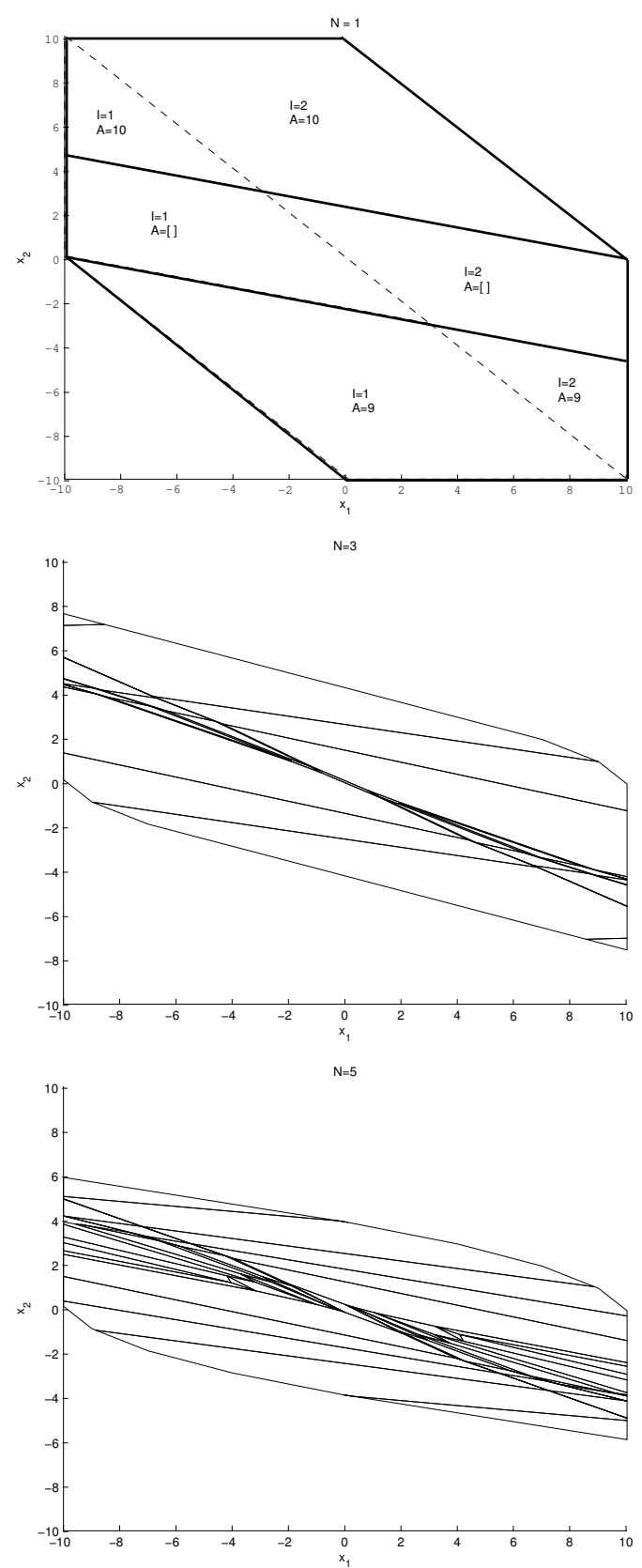

Fig. 1. State space partition of the min-max controller of for system (12) for prediction horizons $\mathrm{N}=1,3,5$.

can be defined using projection algorithms as explained in (Bemporad et al., 2002). Dual degeneracy cannot occur as in the case of general mpQp problems (see (Tøndel et al., 2003)), because as pointed before, the problem is strictly convex. This means that the solver does not need to take into account these degenerate situations.

\section{EXAMPLE}

Consider the double integrator with bounded additive uncertainties

$$
A=\left[\begin{array}{ll}
1 & 1 \\
0 & 1
\end{array}, B=\left[\begin{array}{l}
0 \\
1
\end{array}, D=\left[\begin{array}{c}
0.1 \\
0
\end{array} .\right.\right.\right.
$$



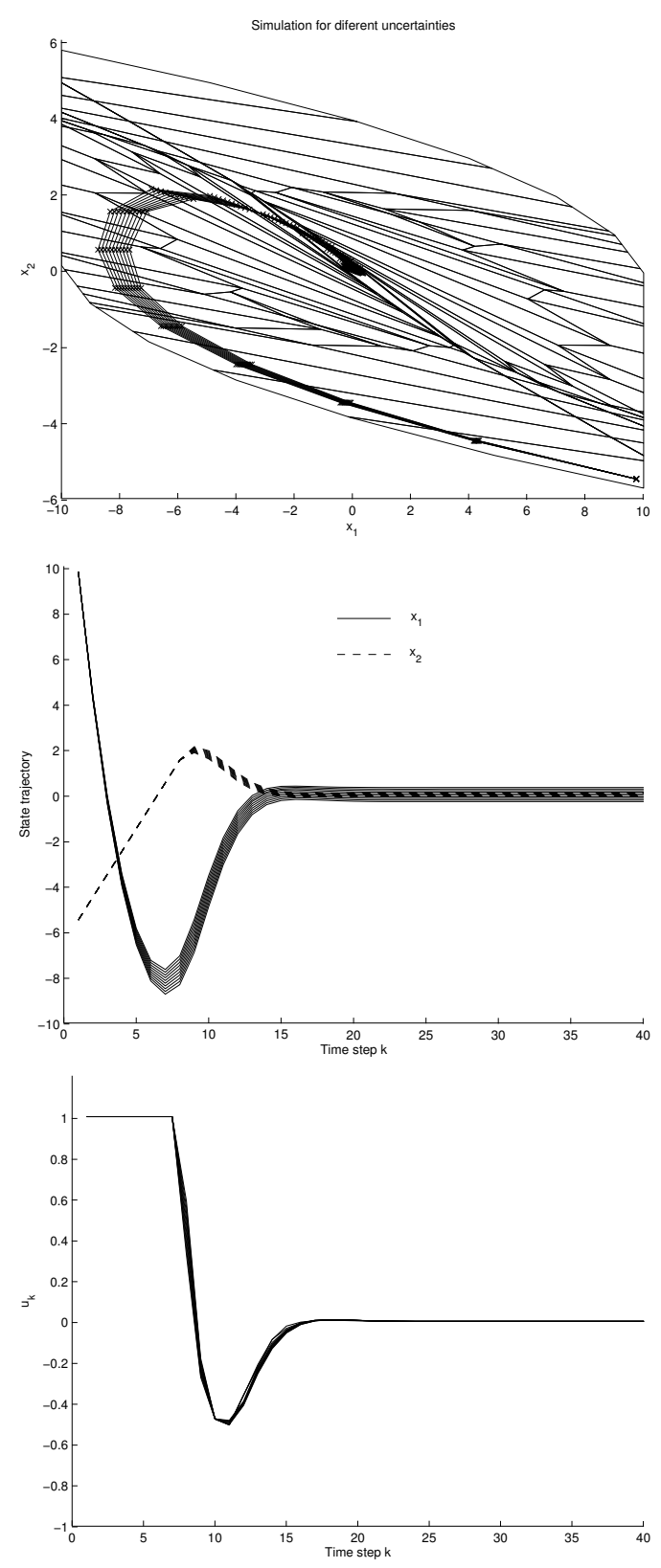

Fig. 2. Simulation results for system (12) with a min-max controller with prediction horizon $\mathrm{N}=8$ for different values of a constant disturbance $w_{k}$.

The uncertainty satisfies $\left\|w_{k}\right\|_{\infty} \leq 1$ and the state and control input are constrained, namely

$$
10 \leq x_{k} \leq 10,-1 \leq u_{k} \leq 1
$$

The control performance objectives are described by

$$
P=Q=\left[\begin{array}{ll}
1 & 0 \\
0 & 1
\end{array}, R=10,\right.
$$

and as linear feedback law is considered the unconstrained LQR control $K=[-0.2054,-0.7835]$.

The critical regions of the resulting controller for prediction horizons of 1,3 , and 5 can be seen in Figure 1. Figure 2 shows simulation results for different constant values of the uncertainty for a controller with a prediction horizon of 8. Table
Table 1. Number of vertices of $W_{N}$ (Ver) and number of critical regions (Reg) for different prediction horizons $(N)$ for system (12).

\begin{tabular}{ccccccc}
$\mathrm{N}$ & 1 & 3 & 5 & 6 & 8 & 10 \\
\hline Ver & 2 & 8 & 32 & 64 & 256 & 1024 \\
Reg & 4 & 45 & 71 & 97 & 147 & 201
\end{tabular}

Table 2. Explicit control law for the min-max controller with prediction horizon $\mathrm{N}=1$ for system (12).

$$
\text { If }\left[\begin{array}{cc}
0.4 & 0.4 \\
1 & 0 \\
1 & 1 \\
-1 & 0 \\
-1 & -1 \\
-0.1 & -0.43 \\
0.1 & 0.43
\end{array}\right] x \leq\left[\begin{array}{c}
0 \\
10 \\
9.9 \\
10 \\
9.9 \\
1 \\
1
\end{array}\right] \text { then }
$$$$
v(0)=\left[\begin{array}{ll}
0.1027 & 0.3463
\end{array}\right] x
$$$$
\text { else if }\left[\begin{array}{cc}
-1 & 0 \\
-2.26 & -9.62 \\
0 & 1 \\
1 & 1
\end{array}\right] x \leq\left[\begin{array}{c}
10 \\
-22 \\
10 \\
9.9
\end{array}\right] \text { then }
$$$$
v(0)=-1+\left[\begin{array}{ll}
0.2054 & 0.7835
\end{array}\right] x
$$$$
\text { else if }\left[\begin{array}{cc}
1 & 0 \\
2.26 & 9.62 \\
0 & -1 \\
-1 & -1
\end{array}\right] x \leq\left[\begin{array}{c}
10 \\
-22 \\
10 \\
9.9
\end{array}\right] \text { then }
$$$$
v(0)=1+\left[\begin{array}{ll}
0.2054 & 0.7835
\end{array}\right] x
$$

else problem is unfeasible

$u(0)=[-0.2054-0.7835] x+v(0)$

1 shows the number of vertices of the polyhedra $W_{N}$ and number of critical regions for different prediction horizons.

Table 2 presents the control algorithm that has to be implemented to obtain the optimum control correction effort $v(0)$ for a prediction horizon of 1 . It can be implemented as a look up table.

As can be seen in Figure 1 the explicit solution for a prediction horizon of 1 consists on six different regions. However, the optimum solution has the same expression in some of them, so it can be unified in only three polyhedral regions of interest that have been remarked in the figure. It can be seen, that where the optimum control law is saturated, the control correction input $v$, has the same gain as the feedback law $K=[-0.2054-$ $0.7835]$, but of inverted sign so the applied input is independent of the state vector. In this figure, vertex $I=1$ and vertex $I=2$ correspond to $w=1$ and $w=-1$ respectively, while constraint $A=9$ and $A=10$ correspond to $u_{0} \geq-1$ and $u_{0} \leq 1$ 
respectively. The dotted line is the region of the state space where the maximum of the optimum solution is attained at $I=[1,2]$ and is a lower dimension region.

The software used to obtain the explicit solutions has been developed in MATLAB. The algorithm implemented is the one proposed in (Tøndel et al., 2003). Although no theoretical result has been obtained, the complexity of the controllers observed in numerous systems is similar to the complexity of a standard MPC explicit control law of the same prediction horizon.

\section{CONCLUSIONS}

The paper has shown that a min-max MPC with a quadratic objective function can be transformed into an mp-QP problem. This allows us to obtain an alternative and more compact proof of the piecewise affine nature of this type of controllers than the one described in literature.

The explicit solution of the min-max not only makes possible the implementation in real plants of these controllers, but also gives an insight of the underlying structure.

\section{REFERENCES}

Alamo, T., D. Muñoz de la Peña, D. Limon and E.F. Camacho (2003). Constrained minmax predictive control: a polynomial time approach. In: Proc. Conference on Decision and Control. Maui, Hawaii, USA.

Allwright, J.C. and G.C. Papavasiliou (1992). On linear programming and robust modelpredictive control using impulse-response. System and Control Letters 18, 159-164.

Bazaraa, M.S. and C.M. Shetty (1979). Nonlinear Programming. Theory and Algorithms. John Wiley \& Sons.

Bemporad, A. (1998). Reducing conservativeness in predictive control of constrained systems with disturbances. In: Proc. American Control Conference. Tampa Florida, USA. pp. 1384-1389.

Bemporad, A., F. Borreli and M. Morari (2003). Min-max control of constrained uncertain discrete-time linear systems. IEEE Trans. Automatic Control 48(9), 1600-1606.

Bemporad, A., M. Morari, V. Dua and E.N. Pistikopoulos (2002). The Explicit Linear Quadratic Regulator for Constrained Systems. Automatica 38, 3-20.

Camacho, E.F. and C. Bordóns (2004). Model Predictive Control in the Process Industry,2nd Edition. Springer-Verlag.
E.C.Kerrigan and J.M. Maciejowski (2004). Feedback min-max model predictive control using a single linear program: Robust stability and the explicit solution. International Journal of Robust and Nonlinear Control 14, 395-413.

H.S.Witsenhausen (1968). A minimax control problem for sampled linear systems. IEEE Transactions on Automatic Control 13(1), 521.

J.H.Lee and Z. Yu (1997). Worst case formulations of model predictive control for systems with bounded parameters. Automatica 3(5), 763781.

Kothare, M.V., V. Balakrishnan and M. Morari (1996). Robust constrained model predictive control using linear matrix inequalities. $A u$ tomatica 32, 1361-1379.

Löfberg, Johan (2003). Minimax Approaches to Robust Model Predictive Control. PhD thesis.

Mayne, D.Q. and S Rakovic (2002). Optimal control of constrained piecewise affine discrete time systems using reverse transformation. In: Proc. Conference on Decision and Control. Las Vegas,Nevada USA. pp. 1546-1551.

Mayne, D.Q., J.B. Rawlings, C.V. Rao and P.O.M. Scokaert (2000). Constrained model predictive control: Stability and optimality. Automatica 36, 789-814.

P.J.Campo and M. Morari (1987). Robust model predictive control. In: Proc. American Control Conference. pp. 1021-1026.

Ramírez, D.R. and E.F. Camacho (2001). On the piecewise linear nature of Min-Max Model Predictive Control with bounded uncertainties. In: Proc. Conference on Decision and Control.

Sakizlis, V., N.M.P. Kakalis, V. Dua, J.D. Perkins and E.N. Pistikopoulos (2004). Design of robust model-based controllers via parametric programming. Automatica 40, 189-201.

Scokaert, P. O. M. and D.Q. Mayne (1998). Min-max feedback model predictive control for constrained linear systems. IEEE Transactions on Automatic Control 43(8), 11361142.

Seron, M.M., G.C. Goodwin and J.A. De Doná (2002). Finitely parameterised implementation of receding horizon control for constrained linear systems. In: Proc. American Control Conference. Anchorage, AK. pp. 4481-4485.

Tøndel, P., T.A. Johansen and A. Bemporad (2003). An algorithm for multi-parametric quadratic programming and explicit MPC solutions. Automatica 39(3), 489-497.

Y.J.Wang and J.B. Rawlings (2003). A new robust model predictive control method I: theory and computation. Journal of Process Control 14(3), 231-247. 